\title{
IgG immunoadsorption in experimental allergic neuritis: effect on antibody levels and clinical course
}

\author{
GRAHAM K HARVEY, * KLAUS SCHINDHELM,† JOHN D POLLARD* \\ From the Department of Medicine, University of Sydney, ${ }^{*}$ and the Centre for Biomedical Engineering, \\ University of New South Wales, $\uparrow$ Kensington, NSW, Australia
}

SUMMARY The effect of IgG immunoadsorption upon the course of chronic experimental allergic neuritis (EAN) is described. Miniature membrane plasma separators coupled with a Protein A (PA)Sepharose immunoadsorbent column were used to perform upon conscious rabbits 5 IgG immunoadsorption treatments over 6 days. Quantitation of anti-myelin IgG and IgM by ELISA revealed that $55-65 \%$ of plasma IgG was removed per treatment. Rapid post-treatment antibody rebound was observed for anti-myelin IgG although no antibody overshoot above control levels could be observed. Anti-myelin IgM levels remained relatively unaffected by PA immunoadsorption. Comparisons of clinical scores between control and treatment animals showed that IgG immunoadsorption was significantly beneficial (day 1 post-treatment $\mathrm{p}<0.001$; day 2 post-treatment $\mathrm{p}<0.05$ ). However, rapid relapse was observed in all treatment animals such that by day 3 post-treatment no significant clinical difference between control and treatment groups could be observed. IgG immunoadsorption suppresses the clinical progression of chronic EAN in a manner similar to that seen with plasma exchange. This finding suggests that antibody modulates early disease pathogenesis.

The results from large controlled clinical trials have established that plasma exchange (PE) is an effective therapy for certain cases of Guillain-Barré syndrome (GBS) ${ }^{1-3}$ However, such trials of PE do not elucidate the mechanisms of disease nor the exact means by which benefit is derived. They do, however, suggest that humoral factors play a role in at least the early disease phase of GBS and leave open the possibility of specific targetting of suspected pathogenic factors such as antibodies.

Protein A immunoadsorption is attracting increasing scrutiny as a technique applicable in a therapeutic setting in humans. The main advantage of IgG immunoadsorption using protein A compared with $\mathrm{PE}$ is that it is a closed loop system which removes a suspected pathogenic factor without the necessity of wasteful discard and replacement of a large proportion of a patient's plasma protein, the majority of which may be normal and fully functional.

In both acute experimental allergic neuritis $(\mathrm{EAN})^{45}$ and chronic $\mathrm{EAN}^{6} \mathrm{PE}$ has been shown to be of clinical

Address for reprint requests: Graham K Harvey, Department of Medicine, University of Sydney, NSW, 2006 Australia.

Received 10 January 1989.

Accepted 6 February 1989 benefit and this further confirms the usefulness of EAN as an experimental model of human peripheral demyelinating neuropathies such as GBS and chronic inflammatory demyelinating polyradiculoneuropathy (CIDP). Because of the potential use for protein A immunoadsorption in a variety of neuromuscular disorders including GBS and CIDP involving suspected pathogenic immunoglobulins, the effects of IgG removal using protein $A$ immunoadsorption were examined in animals with chronic EAN.

\section{Materials and methods}

\section{Induction of EAN}

Female outbred NZW rabbits (mean body weight $2.8 \pm 0.2$ $\mathrm{kg}$ ) were housed in individual cages and given food and water ad libitum. To produce EAN the animals were inoculated with peripheral nervous system myelin prepared from fresh bovine cauda equina using a discontinuous sucrose density gradient centrifugation method similar to that of Norton and Poduslo. ${ }^{78}$ The inoculum consisted of bovine myelin, sterile saline and Freund's complete adjuvant containing $0.5 \mathrm{mg} / \mathrm{ml}$ Mycobacterium tuberculosis (Commonwealth Serum Laboratories, Melbourne, Australia) mixed in the ratio of $1 \mathrm{~g}: 1 \mathrm{ml}: 2 \mathrm{ml}$ respectively and homogenised (Polytron, Kinematica). Under halothane/oxygen anaesthesia each animal received a single subcutaneous multiportal inoculation consisting of $0.25 \mathrm{ml}$ to each paw and $0.25 \mathrm{ml}$ to two separate sites on the back. 
Table 1 Clinical assessment of EAN

\begin{tabular}{ll}
\hline Grade & Clinical disability \\
\hline 0 & Normal \\
1 & Slight postural abnormality, less lively \\
2 & Mild but definite paraparesis \\
3 & Moderate paraparesis, possible front leg weakness \\
4 & Tetraparesis \\
5 & Severe tetraparesis, still ambulatory \\
6 & Severe tetraparesis, non-ambulatory, respiratory \\
& difficulty, possible incontinence \\
7 & Moribund or dead \\
\hline
\end{tabular}

Clinical assessment and blood sampling

Animals were periodically weighed and examined for signs of EAN and a clinical disability score assigned according to the classifications in table 1. Animals were scored independently by two observers during each clinical examination.

Two ml blood samples were taken by venepuncture from peripheral ear veins into sequestrene tubes and centrifuged at $500 \mathrm{~g}$ for 10 minutes. Plasma samples were stored at $-70^{\circ} \mathrm{C}$.

\section{Blood access}

Blood access was obtained via an externalised carotidjugular arteriovenous shunt inserted under halothane/ oxygen anaesthesia as described previously. ${ }^{6}$ Control animals underwent a sham operation under halothane/oxygen anaesthesia in which the right carotid artery and jugular vein were isolated and ligated.

\section{Extracorporeal circuit}

The extracorporeal circuit is depicted in fig 1 . The membrane plasma separators were of the hollow fibre type made of polypropylene (Enka AG, Wuppertal, FRG). The filters had an effective surface area of $200 \mathrm{~cm}^{2}$ with a maximum pore size of $0.5 \mu \mathrm{m}$. The blood side filter priming volume was approximately $2 \mathrm{ml}$. The immunoadsorption column was constructed using PA-Sepharose CL-4B (Pharmacia, Uppsala, Sweden) swollen and packed between $30 \mu \mathrm{m}$

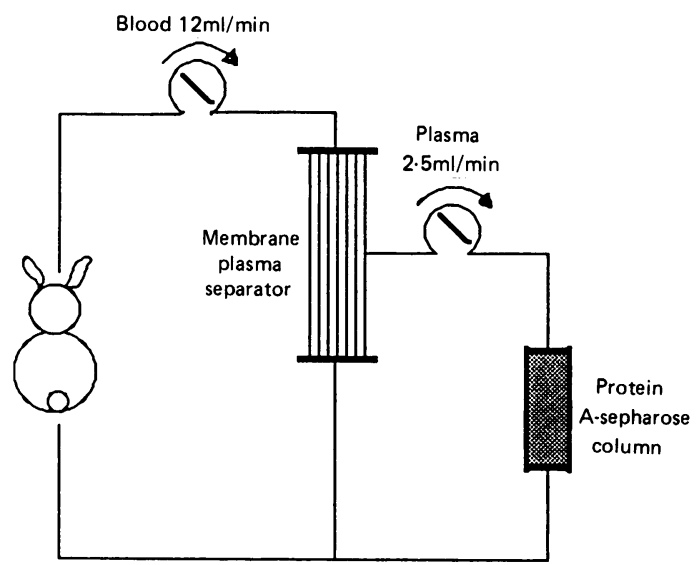

Fig 1 Schematic diagram of extracorporeal immunoadsorption system. polyethylene supports in a $25 \mathrm{ml}$ glass column with a length/ diameter ratio of $6: 1$. Based upon the known IgG binding capacity sufficient PA-Sepharose was packed to enable a total binding capacity of approximately $500 \mathrm{mg}$ representing at least $50 \%$ of the circulating plasma IgG in the rabbit. This column capacity for IgG has been confirmed previously. ${ }^{9}$ The column void volume was approximately $15 \mathrm{ml}$. Blood and plasma flow rates were regulated by a multi-channel peristaltic pump (Gilson Medical, Paris, France). Blood flow through the filter was maintained at $12 \mathrm{ml} / \mathrm{min}$ while plasma flow through the column was maintained at $2.5 \mathrm{ml} / \mathrm{min}$ enabling a plasma residence time within the gel of approximately 6 minutes. Total extracorporeal blood circuit volume was approximately $15 \mathrm{ml}$.

\section{Immunoadsorption procedures}

As animals reached a clinical grade of 2-3 they were paired and randomly allocated into control and treatment groups. Immediately after allocation the treatment animals received an externalised arteriovenous shunt and control animals underwent a sham operation.

Before each immunoadsorption procedure the extracorporeal circuit was primed with $0 \cdot 15 \mathrm{M}$ sterile saline. Animals were lightly restrained by wrapping in a surgical drape and each remained conscious and calm throughout the procedure. Just prior to connection to the circuit, rabbits received $1500 \mathrm{U}$ heparin intravenously and this was allowed to distribute within the intravascular space. A pre-treatment blood sample was taken and the arterial extracorporeal line leading to the filter was then connected to the shunt and the blood circuit primed under arterial pressure to expel residual saline. When the blood circuit was complete the venous extracorporeal line was connected to the shunt and the pump started. The first $20 \mathrm{ml}$ of saline effluent from the column were discarded after which time the post-column plasma line was reconnected to allow treated plasma to be returned to the animal. Column flow rate was measured prior to reconnection and the time necessary to process 1 plasma volume (30 $\mathrm{ml} / \mathrm{kg}$ ) calculated. After processing 1 plasma volume the peristaltic pump was stopped and the arterial line disconnected from the shunt. The extracorporeal circuit was then flushed with sterile saline to return the contents to the animal. Similarly, the column was flushed with $25 \mathrm{ml}$ of sterile saline returning unbound column contents to the animal. The venous line was then disconnected from the animal and arterial and venous shunt cannulae were reconnected behind the ear. After a brief period (approximately 5 minutes) to allow vascular mixing, a post-treatment blood sample was taken.

The PA-Sepharose column was regenerated between immunoadsorption treatments by desorption with at least 3 column volumes of $0.1 \mathrm{M}$ glycine- $\mathrm{HCl}(\mathrm{pH} \mathrm{2.8)}$ and then equilibrated with sterile saline.

Treatment animals underwent immunoadsorption procedures five times within 6 days. Each was treated daily for the first 3 days followed by a day with no treatment and then the final two treatments were conducted on the 5th and 6 th days.

Measurement of anti-myelin $\operatorname{Ig} G$ and $\operatorname{Ig} M$

Anti-myelin IgG and IgM were quantitated using an ELISA assay which has been previously described in detail. ${ }^{8}$ 
Measurement of biochemical and haematological parameters Biochemical analyses were conducted on an American Monitor Parallel autoanalyser and haematological analyses were conducted using a Technicon $\mathrm{H} 1$ blood analyser.

\section{Results}

\section{Clinical features}

Out of a total of 20 animals inoculated with myelin, 17 animals were allocated into either control or treatment groups. In these animals onset of neurological signs of EAN occurred between days 12 and 16 post-inoculation. Three animals showed an EAN disease of late onset, one on day 23 and two on day 40 postinoculation and were not included in the study. After pairing and random allocation, eight animals were assigned to the treatment group and nine to the control group. Mean clinical score at allocation for the control group was 2.56, SD 0.53 and for the treatment group $2 \cdot 57$, SD 0.53 . One treatment animal died between the first and second immunoadsorption procedures from catastrophic shunt uncoupling and was not included in the final study.

Clinical data are depicted in fig 2. Data are displayed as arithmetic changes in clinical scores from the scores at allocation. Control animals showed a severe progressive neuropathy with one animal becoming moribund on day 44 post inoculation and two showing severe tetraparesis with respiratory difficulty on days 47 and 55 post-inoculation. Animals becoming moribund were immediately killed. Animals attaining a score of 6, that is, severe quadriparesis and respiratory difficulty (with possible incontinence) that did not show improvement after 2 days were killed and included in the data as attaining a score of 7.

In contrast to the progressive disease seen in the control group, treatment animals showed significant

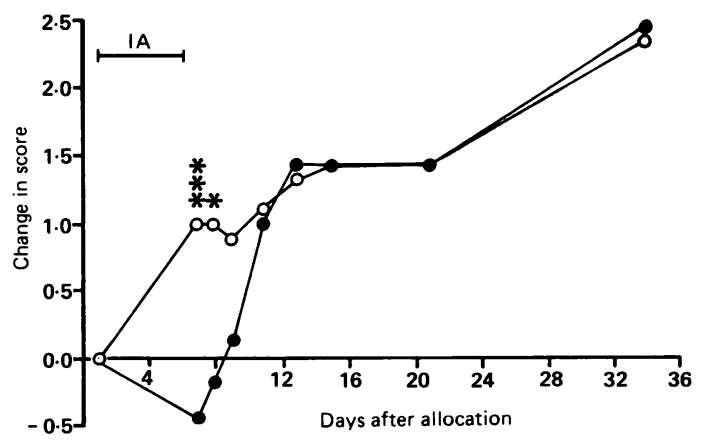

Fig 2 Mean changes in clinical score after allocation for the control group (open circles, $n=9$ ) and treatment group (closed circles, $n=7$ ). Bar indicates period of treatment. Significant differences were determined by two-sided Mann-Whitney U-tests; $<<0.001^{* * *} ; p<0.05^{*}$.
Table 2 Changes in blood components after a single immunoadsorption

\begin{tabular}{lll}
\hline Component & $\%$ Change (Mean $\mathrm{SD}, n=5)$ \\
\hline $\mathrm{Na}, \mathrm{K}, \mathrm{Cl}, \mathrm{Ca}, \mathrm{PO}$, & no change & - \\
Albumin & $-13.79,6.42$ & $\mathrm{p}<0.05$ \\
Total protein & $-19 \cdot 08,7.45$ & $\mathrm{p}<0.01$ \\
Haematocrit & no change & - \\
Red cell count & no change & - \\
White cell count & $-47.58,11 \cdot 11$ & $\mathrm{p}<0.01$ \\
\hline
\end{tabular}

clinical remission as a result of IgG immunoadsorption. Statistical analysis of data taken directly after the course of treatments (days 7 and 8 post-allocation) using 2-tailed Mann-Whitney U tests showed significant differences between the two groups ( $p<$ $0.001 ; p<0.05$ respectively). Remission was transient and most animals showed a relapse by days 9 to 11 post-allocation.

\section{Blood biochemistry and haematology}

Changes in blood composition from a single immunoadsorption are shown in table 2. Percentage changes in haematological parameters were calculated using blood samples taken from five animals before and directly after the first immunoadsorption procedure. There was no change in plasma solutes, haematocrits or erythrocyte counts. The lack of effect upon haematocrits reflected minimal alterations in plasma volume. However, immunoadsorption did result in significant decreases in albumin and total protein concentrations and white cell counts. Changes in white cell counts have been previously reported in other forms of extracorporeal therapy such as haemodialysis. $^{10}$ Total protein concentration was reduced by approximately $20 \%$ due mainly to IgG removal. The slight but significant decrease in albumin concentration associated with the extracorporeal procedures was due to haemodilution resulting from the return of blood in the venous line with saline and to a lesser extent nonspecific protein binding within the gel matrix.

\section{Plasma anti-myelin IgG and IgM}

Anti-myelin IgG and IgM levels were assessed in both groups and are depicted in figure 3. Antibody levels are displayed normalised to a pre-treatment level of $100 \%$. Anti-myelin IgG levels rose steadily in the control group throughout the period of observation. In the treatment group, each IgG immunoadsorption decreased circulating anti-myelin IgG concentrations by $55-65 \%$. Rapid post-treatment antibody rebound was observed after each procedure so that levels were re-established within 24 hours. However, the course of immunoadsorption treatments held anti-myelin IgG concentrations at pre-treatment levels in the treatment group so that at day seven post-allocation there were 

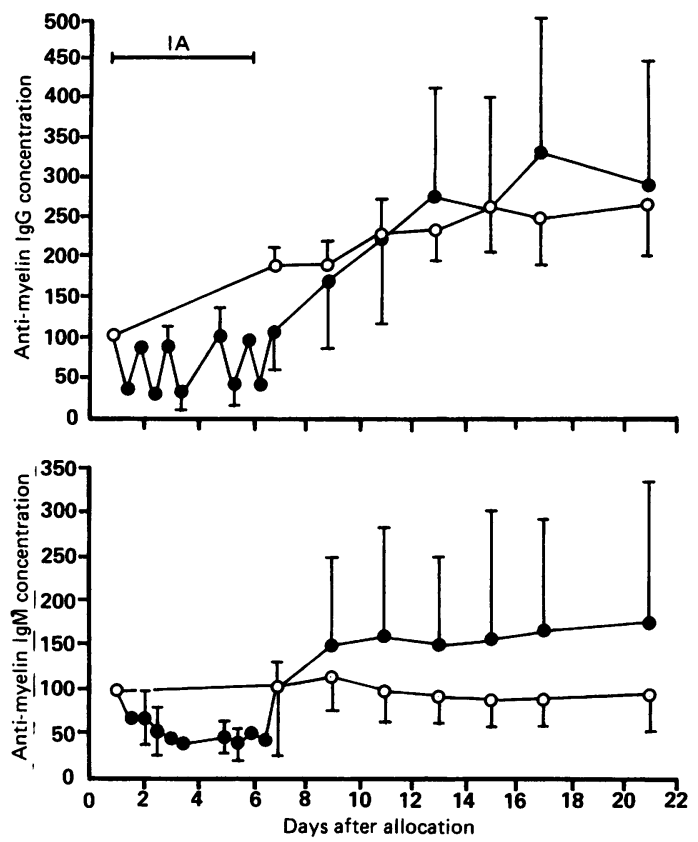

Fig 3 Plasma anti-myelin IgG and IgM concentrations (arbitrary mass units) determined from samples obtained up to 21 days post allocation. Data are normalised to a pre-treatment level of $100 \%$. Mean, SD is displayed for the control group (open circles, $n=9$ ) and treatment group (closed circles, $n=7$ ). Bar indicates period of treatment.

significant differences in anti-myelin IgG concentrations between control and treatment groups (2-tailed Student's $t$ test, $\mathrm{p}<0.001$ ). Anti-myelin IgG levels quickly increased after cessation of treatment so that two days later, that is, day nine post-allocation there was no significant difference in levels between groups.

Although rapid antibody rebound was observed, there was no evidence of antibody overshoot above control levels as a result of transient removal. The level in the treatment group was still identical to the control level when last measured at day 20 post-allocation.

Anti-myelin IgM levels are also shown in figure 3 normalised to a pre-treatment level of $100 \%$. In the control group, levels were constant throughout the period of observation. In the treatment group, antimyelin IgM levels appeared to decrease slowly during treatment although comparisons of pre- and postimmunoadsorption concentrations showed no differences and suggest no significant removal via specific binding to protein $\mathrm{A}$. The slight decreases in anti-myelin IgM concentrations could be due to some entrainment in the PA-Sepharose column. Although after the course of immunoadsorption procedures anti-myelin IgM levels appeared to increase above control levels, statistical analysis using 2-tailed Student's $t$ test showed no significant differences.

\section{Discussion}

The results of this study demonstrate that a short intensive course of IgG immunoadsorption removing $55-65 \%$ of circulating IgG is sufficient to induce clinical remission during the early disease phase of EAN. The observed benefit in this study complements that previosly seen in EAN with the use of membrane PE. ${ }^{6}$ The significant but temporary nature of remission is undoubtedly due to the severe chronic progressive type of disease of which the clinical, electrophysiological and histological features have been well documented." In both therapeutic studies assessment of clinical disability directly after the course of treatments showed significant clinical benefit. This was followed by rapid relapse within one week so that animals receiving a single course of treatment were in the long term clinically indistinguishable from controls. The situation is therefore similar to that in CIDP in man where the need for repeated exchanges to maintain benefit is well recognised,,$^{12}$ although in most cases the period between exchanges is somewhat longer. ${ }^{13}$

The early disease phase in EAN in these animals is characterised by weight loss and mild to moderate paraparesis. At this stage detectable levels of antimyelin IgG are present and show gradual increases during disease progression. High levels of anti-myelin IgM can also be detected in some but not all animals. ${ }^{8}$ The concomitant increases in clinical disability with increases in anti-myelin IgG combined with the ability of IgG removal alone to mimic the clinical remission observed in EAN with membrane $\mathrm{PE}^{6}$ and centrifugation $\mathrm{PE}^{45}$ suggest a pathogenic role for $\mathrm{IgG}$ antibodies. Intraneural transfer experiments involving whole EAN serum have demonstrated humoral factors which cause demyelination and conduction failure ${ }^{14}$ but although these experiments may detect the presence of pathogenic humoral factors, they fail to determine their nature. Anti-myelin activity of whole rabbit EAN serum has been demonstrated in organotypic PNS cultures to be complement dependent ${ }^{15}$ suggesting antibody involvement. Rat intraneural injection studies have clearly shown advanced myelin and Schwann cell damage with rabbit EAN serum well before a macrophage presence ${ }^{16}$.

Intraneural injection of GBS serum into rat sciatic nerves also induces demyelination ${ }^{17-20}$ although the severity of effect is often less than with EAN serum. Recent detailed sequential morphometric studies confirm the ability of GBS serum to demyelinate peri- 
pheral nerves in vivo in the absence of cells. ${ }^{21}$ The presence of anti-nerve antibodies in GBS serum has been described ${ }^{22-25}$ and correlates with response to $\mathrm{PE}^{24}$ Levels appear to correlate with disease severity and are at a peak during the early disease phase. ${ }^{2627}$ These observations along with results from large controlled trials showing that therapeutic benefit in GBS is derived from PE only if used early in the disease course $^{1-3}$ strongly suggest a causative role for antinerve immunoglobulins.

This study demonstrates the ability of IgG immunoadsorption to produce clinical remission during the early disease phase of EAN. Although PE has been used extensively in the treatment of human as well as experimental diseases, therapeutic immunoadsorption is still in its infancy. Immunochemical adsorbents have long been utilised for routine in vitro separation but Terman et al $^{28}$ and Bansal et al ${ }^{29}$ were the first to incorporate affinity chromatographic techniques and plasma separation technology to achieve ex vivo on line adsorption of specific plasma proteins.

Bansal et al $^{30}$ first described the successful treatment of colon carcinoma using a protein A-carrying immunoadsorbent perfused with patient's plasma. This study utilised the discovery of the known capacity of protein A to bind IgG. ${ }^{31}$ Since then, with more widespread use of extracorporeal systems, specific haemodynamic effects have been attributed to the use of extracorporeal therapy. The use of membranes for plasma separation generally induces transient decreases in white cell counts. ${ }^{32}$ This effect has been clearly measured in our study. Such decreases are thought to be the result of complement activation and leukocyte sequestration in the pulmonary vascular bed. ${ }^{1033}$ The binding of IgG to protein $A$ forms an immune complex which may activate the classical complement pathway ${ }^{34}$ although this effect was not reported in more recent studies. ${ }^{35}$

In our study the level of complement activation and the metabolic fate of activated cascade proteins have not been measured. McLeod et $a^{32}$ reported a C3 conversion of $8-15 \%$ upon plasma contact with polypropylene membranes. Charlton ${ }^{36}$ measured transient decreases in rabbit $\mathrm{C} 3$ concentrations of approximately $20 \%$ with the use of polypropylene membrane plasma separators. In this study there were no further decreases in $\mathrm{C} 3$ levels as a result of exposure to protein A-Sepharose suggesting minimal complement activation from the immune complexing of IgG to protein A. The extent to which complement activation contributed to or attenuated the clinical remission observed during early phase EAN is not known. It is highly improbable that transient complement activation alone of the magnitude likely in this present study could account for the marked abatement of disease progression observed in treatment animals. Such animals showed no apparent signs of lung congestion or bronchospasms. Although such symptoms were noted by Bansal et al ${ }^{30}$ and Terman ${ }^{37}$ in patients treated with protein A-carrying staphylococcus immunoadsorbents, these were probably attributable to the use of a bacterial product in place of the purified protein A used in our study.

Of more concern is the possible clinical effect of endogenous steroid synthesis in the treated animals. In the present study, control animals underwent a sham operation but no sham immunoadsorption treatments. There exists the possibility that steroid production in treated animals may have had a positive clinical effect over untreated control animals. Studies have shown both prophylactic and therapeutic effects of steroids upon EAN ${ }^{38-40}$ although earlier experiments were unable to detect significant effects. ${ }^{41}$ In a previous study examining the effects of membrane PE upon chronic EAN, ${ }^{6}$ plasma cortisol levels were determined in all control and treated animals. The results showed no significant lasting effects of sham operations and shunt insertions upon plasma cortisol concentrations. Moreover, analysis of plasma cortisol levels before and after PE in the treated animals showed a decrease attributable to plasma removal exactly as predicted from a steady state single pool model and no significant increases in endogenous cortisol synthesis during treatment. The lack of effect of membrane PE upon plasma steroid concentration would strongly suggest a typical lack of effect in the similarly treated but selectively IgG depleted animals of the present study.

A major drawback of therapeutic PE is the need to use substitute fluids such as albumin or fresh frozen plasma with the concomitant expense and accompanying side effects. Extracorporeal immunoadsorption promises to be a viable alternative in diseases with identified circulating pathogenic material and as a valuable experimental tool for elucidating speculative roles for plasma components such as antibodies. A trial of IgG immunoadsorption in acute phase GBS seems appropriate. The results of experimental trials of PE and IgG immunoadsorption in EAN together with accumulating evidence for a role for antibodies in GBS and CIDP in initiating or augmenting the early disease process suggest that the specific targetting of possible effectual agents such as antibodies remains a rational and relevant therapeutic approach.

The assistance of Enka AG, Wuppertal, FRG is gratefully acknowledged. This study was supported by the National Health and Medical Research Council of Australia.

\section{References}

1 Osterman PO, Fagius J, Lundemo G, et al. Beneficial effects of plasma exchange in acute inflammatory polyradiculoneuropathy. Lancet 1984;ii:1296-9. 
2 The Guillain-Barré Study Group. Plasmapheresis and acute Guillain-Barré syndrome. Neurology 1985;35:1096-104.

3 French Cooperative Group on Plasma Exchange in Guillain-Barré Syndrome. Efficiency of plasma exchange in Guillain-Barré syndrome: role of replacement fluids. Ann Neurol 1987;22: 753-61.

4 Antony JH, Pollard JD, McLeod JG. Effects of plasmapheresis on the course of experimental allergic neuritis in rabbits. J Neurol Neurosurg Psychiatry 1981;44:1124-8.

5 Gross MLP, Craggs RI, King RHM, Thomas PK. The treatment of experimental allergic neuritis by plasma exchange. $J$ Neurol Sci 1983;61:149-60.

6 Harvey GK, Schindhelm K, Antony JH, Pollard JD. Membrane plasma exchange in experimental allergic neuritis: effect on antibody levels and clinical course. $J$ Neurol Sci 1988;88:207-18.

7 Norton WT, Poduslo SE. Myelination in rat brain: method of myelin isolation. $J$ Neurochem 1973;21:749-57.

8 Harvey GK, Schindhelm K, Antony JH, Pollard JD. Linear response curves from an ELISA assay: measurement of antimyelin IgG and IgM during experimental allergic neuritis. $J$ Neurosci Meth 1987;21:81-90.

9 Charlton B, Schindhelm K, Farrell PC. Selective adsorption of IgG and IgM: evaluation of systems for plasma exchange. Trans Am Soc Artif Intern Organs 1982;28:400-3.

10 Craddock PR, Fehr J, Dalmasso AP, Brigham KL, Jacob HS. Haemodialysis leukopenia. Pulmonary vascular leukostasis resulting from complement activation by dialyzer cellophane membranes. J Clin Invest 1977;59:879-88.

11 Harvey GK, Pollard JD, Schindhelm K, Antony J. Chronic experimental allergic neuritis. An electrophysiological and histological study in the rabbit. J Neurol Sci 1987;81:215-25.

12 Pollard JD, McLeod JG, Gatenby P, Kronenberg H. Prediction of response to plasma exchange in chronic relapsing polyneuropathy. J Neurol Sci 1983;58:269-87.

13 Pollard JD. A critical review of therapies in acute and chronic inflammatory demyelinating polyneuropathies. Muscle Nerve 1987;10:214-21.

14 Saida K, Saida T, Brown MJ, Silberberg DH, Asbury AK. Antiserum-mediated demyelination in vivo: a sequential study using intraneural injection of experimental allergic neuritis serum. Lab Invest 1978;39:449-62.

15 Raine CS, Bornstein MB. Anti-myelin activity of EAN serum in organotypic PNS/CNS cultures (Abstr). J Neuropathol Exp Neurol 1978;37:676.

16 Hahn AF, Gilbert JJ, Feasby TE. Passive transfer of demyelination by experimental allergic neuritis serum. Acta Neuropathol 1980;49:169-76.

17 Feasby TE, Hahn AF, Gilbert JJ. Passive transfer of demyelinating activity in Guillain-Barré polyneuropathy (Abstr). Neurology 1980;30:363.

18 Cook SD, Dowling PC. The role of autoantibody and immune complexes in the pathogenesis of Guillain-Barré syndrome. Ann Neurol 1981;(suppl 9):70-9.

19 Saida T, Saida K, Lisak RP, Brown MJ, Silberberg DH, Asbury AK. In vivo demyelinating activity of sera from patients with Guillain-Barré syndrome. Ann Neurol 1982;11:69-75.

20 Harrison BM, Hansen LA, Pollard JD, McLeod JG. Demyelination induced by serum from patients with Guillain-Barré syndrome. Ann Neurol 1984;15:163-70.

21 Brown MJ, Rosen JL, Lisak RP. Demyelination in vivo by Guillain-Barré syndrome and other human serum. Muscle Nerve 1987;10:263-71.

22 Lisak RP, Zwiman B, Norman M. Antimyelin antibodies in neurologic diseases. Arch Neurol 1975;32:163-7.

23 Nyland H, Aarli JA. Guillain-Barré syndrome: Demonstration of antibodies to peripheral nerve tissue. Acta Neurol Scand 1978; 58:35-43.

24 Vedeler CA, Nyland H, Fagius J, et al. The clinical effect and the effect on serum IgG antibodies to peripheral nerve tissue of plasma exchange in patients with Guillain-Barré syndrome. Neurology 1982;228:59-64.

25 Ryberg B. Extra- and intrathecal production of antinerve and antibrain antibodies in Guillain-Barré syndrome: evaluation by an antibody index. Neurology 1984;34:1378-81.

26 Koski CL, Humphrey R, Shin ML. Anti-peripheral myelin antibody in patients with demyelinating neuropathy: Quantitation and kinetic determination of serum antibody by complement component 1 fixation. Proc Natl Acad Sci 1985;82:905-9.

27 Koski CL, Gratz E, Sutherland J, Mayer RF. Clinical correlation with anti-peripheral-nerve myelin antibodies in Guillain-Barré syndrome. Ann Neurol 1986;19:573-7.

28 Terman DS, Petty D, Ogden D, Pefley C, Buffaloe G. Specific extraction of antigen in vivo by extracorporeal circulation over antibody immobilized in collodion-charcoal. J Immunol 1976; 117:1971-5.

29 Bansal SC, Bansal BR, Rhoads JE Jr, Cooper DR, Borland JP, Mark R. Ex-vivo removal of mammalian immunoglobulin G: method and immunological alterations. Int J Artif Organs 1978; 1:94-103.

30 Bansal SC, Bansal BR, Thomas HL, et al. Ex vivo removal of serum IgG in a patient with colon carcinoma: some biochemical, immunological and histological observations. Cancer 1978;42 $1-18$

31 Jensen K. Investigations of staphylococcal antigen structure, with special reference to a polysaccharide antigen and normally present antibodies against this antigen. Doctoral thesis, Munksgaard, Copenhagen, 1959.

32 McLeod BC, Viernes A, Sassetti RJ. Complement metabolism during membrane plasma separation. Artif Organs 1983;7: 443-9.

33 Hammerschmidt DE, Craddock PR, McCullough J, Kronenberg RS, Dalmasso A, Jacob HS. Complement activation and pulmonary leukostasis during nylon fiber filtration leukapheresis. Blood 1978;51:721-30.

34 Stalenheim G, Götze O, Cooper NR, Sjoquist J, Muller-Eberhard HJ. Consumption of human complement components by complexes of $\mathrm{IgG}$ with protein A of Staphylococcus aureus. Immunochem 1973;10:501-7.

35 Nilsson IM, Jonsson S, Sundqvist SB, Ahlberg A, Bergentz S-E. A procedure for removing high titer antibodies by extracorporeal protein-A-sepharose adsorption in hemophilia: substitution therapy and surgery in a patient with hemophilia B and antibodies. Blood 1981;58:38-44.

36 Charlton B. Extracorporeal immunoadsorption: a study of applications. Doctoral thesis, University of New South Wales, Australia, 1985.

37 Terman DS. Plasma perfusion over immobilized protein A from Staphylococcus aureus for treatment of cancer: observations on the evolution of plasma perfusion systems. Int $J$ Artif Organs 1982;5:77-80.

38 Hughes RAC, Kadlubowski M, Hufschmidt A. Treatment of acute inflammatory polyneuropathy. Ann Neurol 1981;9(suppl): 125-33.

39 King RHM, Craggs RI, Gross MLP, Thomas PK. Effects of glucocorticoids on experimental allergic neuritis. Exp Neurol 1985;87:9-19.

40 Feasby TE, Gilbert JJ, Hahn AF, Lovgren DS. Treatment of experimental allergic neuritis with prednisolone. Can J Neurol Sci 1987; 14:46-9.

41 Heitmann R, Mannweiler K. Experimental animal studies on allergic polyneuritis. Deut Zschr Nervenh 1957;177:28-47. 